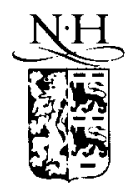

FI SHVIFIR

\title{
Bounding the tau neutrino magnetic moment from single photon searches at LEP
}

\author{
Thomas M. Gould ${ }^{\mathrm{a}, 1}$, I.Z. Rothstein ${ }^{\mathrm{b}, 2}$ \\ a Department of Physics and Astronomy, The Johns Hopkins University, Baltimore, MD 21218, USA \\ b The Randall Laboratory of Physics, University of Michigan, Ann Arbor, MI 48109, USA \\ Received 25 May 1994 \\ Editor: H. Georgi
}

\begin{abstract}
We show that single photon searches at LEP constrain the tau neutrino magnetic moment to be less than $\mathcal{O}\left(10^{-6}\right) \mu_{B}$. This bound is competitive with low energy $(\sqrt{s} \simeq 30 \mathrm{GeV})$ single photon searches.
\end{abstract}

The present bounds on the electromagnetic properties of the tau neutrino are several orders of magnitude worse than the bounds on the electron and muon neutrinos. To date, the best bounds on tau neutrino electromagnetic properties have been derived from single photon searches at $e^{+} e^{-}$colliders below the $Z$ resonance, $\sqrt{s} \simeq 30 \mathrm{GeV}$ (e.g. MAC, ASP, CELLO, Mark-J) [1]. In this short note, we point out that comparable constraints on the tau neutrino magnetic moment can be obtained from single photon searches at LEP. (For a previous estimate, see [2].) These bounds are relevant for ruling out the possibility of the tau neutrino having a mass in the $\mathrm{MeV}$ range $[3,4]$.

Single photon searches at $\mathrm{L} 3$ have provided a measurement of the number of neutrino species, as well as bounds on the couplings of heavy $\left(m_{\nu} *>43 \mathrm{GeV}\right)$ "excited" neutrino to electroweak gauge particles $\left(E_{\gamma}>10 \mathrm{GeV}\right)$ [5], and the compositeness of the $Z$ and the mass of light gravitinos $\left(E_{\gamma}>\frac{1}{2} E_{\text {beam }}\right)$ [6]. Single photon searches at ALEPH have also provided

1 gould@fermi.pha.jhu.edu.
2 irothstein@umiphys.bitnet. a measurement of the number of neutrino species, as well as bounds on the couplings of heavy "excited" neutrino to electroweak gauge particles [7]. Rizzo has derived constraints on anomalous $Z \nu \bar{\nu}$ couplings from the L3 measurement of the $Z$ width [8]. In a comprehensive approach, Escribano and Massó have recently derived constraints on all electromagnetic fermion couplings from LEP-I data [9]. We show below that the LEP searches for single energetic photons, with no other particles detected, also provide a strong accelerator-based constraint on the tau neutrino magnetic moment.

Possible electromagnetic properties of a massive Dirac neutrino are summarized in the current

$$
J_{\mu}=e \bar{\nu}\left(p^{\prime}\right)\left(F_{1}\left(q^{2}\right) \gamma_{\mu}+i \frac{F_{2}\left(q^{2}\right)}{2 m_{\nu}} \sigma_{\mu \nu} q^{\nu}\right) \nu(p),
$$

with $F_{1,2}\left(q^{2} \equiv\left(p^{\prime}-p\right)^{2}\right)$ dimensionless structure functions. The magnetic moment $\kappa$ is

$\kappa \mu_{B}=\frac{e F_{2}(0)}{2 m_{\nu}}$, 
in units of the Bohr magneton $\mu_{B}$. Single photon searches probe the form factor at zero momentum transfer, obviating off-shell extrapolations.

At low center of mass energy $s \ll M_{z}^{2}$, the dominant contribution to the process

$e^{+} e^{-} \rightarrow \nu \bar{\nu} \gamma$

involves the exchange of a virtual photon [1]. Dependence on the magnetic moment comes from a direct coupling to the virtual photon, and the observed photon is a result of initial state Bremsstrahlung. At higher $s$, near the $Z$ pole $s \simeq M_{z}^{2}$, the dominant contribution for $E_{\gamma}>10 \mathrm{GeV}$ involves the exchange of a $Z$. Dependence on the magnetic moment now comes from the final state radiation of the observed photon. We emphasize here the importance of final state radiation near the $Z$ pole, which occurs preferentially at high $E_{\gamma}$ compared to conventional Bremsstrahlung. It has been previously believed that physics dominated by the $Z$ pole is insensitive to electromagnetic properties [1].

The magnetic moment coupling gives a contribution to the differential cross-section for the process $e^{+} e^{-} \rightarrow \nu \bar{\nu} \gamma$ of the form

$\frac{d \sigma}{E_{\gamma} d E_{\gamma} d \cos \theta_{\gamma}}=\frac{\alpha^{2} \kappa^{2}}{96 \pi} \mu_{B}^{2} C\left[x_{w}\right] F\left[s, E_{\gamma}, \cos \theta_{\gamma}\right]$,

where $E_{\gamma}, \cos \theta_{\gamma}$ are the energy and scattering angle of the photon. The kinematics are contained in the function

$F\left[s, E_{\gamma}, \cos \theta_{\gamma}\right] \equiv \frac{s-2 \sqrt{s} E_{\gamma}+\frac{1}{2} E_{\gamma}^{2} \sin ^{2} \theta_{\gamma}}{\left(s-M_{z}^{2}\right)^{2}+M_{z}^{2} \Gamma_{z}^{2}}$.

The coefficient $C$ is

$\mathcal{C}\left[x_{w}\right] \equiv \frac{8 x_{w}^{2}-4 x_{w}+1}{x_{w}^{2}\left(1-x_{w}\right)^{2}}$,

using Standard Model $Z e \vec{e}$ and $Z \nu \bar{\nu}$ couplings, and $x_{w} \equiv \sin ^{2} \theta_{w}$. In using (3), we neglect initial state radiation, and $W$ and photon exchange graphs, which amount to $1 \%$ corrections in the relevant kinematic regime.

Now we constrain the magnetic moment by combining some LEP data sets, with different integrated luminosities $L$, cuts on photon angle and energy. There
Table 1

\begin{tabular}{lrrrllll}
\hline Exp. & & $L\left\{\mathrm{pb}^{-1}\right\}$ & $\theta_{\min }, \theta_{\max }$ & $E_{\min }\{\mathrm{GeV}\}$ & Events & Ref. \\
\hline L3 & 1990 & 3.8 & 45,135 & 10 & 0 & {$[5]$} \\
& 1991 & 11.2 & 45,135 & 22.8 & 0 & {$[6]$} \\
ALEPH & 1991 & 8.5 & 42,138 & 17 & 0 & {$[7]$} \\
\hline
\end{tabular}

were no events allowed by the cuts in any of the experiments.

Integrating (3) over the relevant range for each experiment, we obtain estimates of the number of single photon events. The combined number of events expected at LEP due to a neutrino magnetic moment is

$N \simeq \times 7.210^{10} \kappa^{2}$.

from data at the $Z$ resonance only. Using Poisson statistics, we require (6) be less than 2.2 , giving a bound on the magnetic moment at the $90 \%$ confidence level. This implies a bound

$\kappa \lesssim 5.5 \times 10^{-6}$ at $90 \%$ C.L.

This result compares favorably with the bounds obtained from low-energy single photon searches $(4.0 \times$ $10^{-6}$ at $90 \%$ C.L.) [1] and from LEP-I data on $Z$ partial widths $\left(3.6 \times 10^{-6}\right.$ at $68 \%$ C.L.) [9]. The derived bound (7) could be improved by including data from the entire $Z$ resonance and may supercede the best bound in [9]. We estimate that the inclusion of the full integrated luminosity for each experiment can at best improve the bound by $\sqrt{2}$, giving

$\kappa \lesssim 3.9 \times 10^{-6}$ at $90 \%$ C.L.

We expect a more careful analysis of the data will yield a bound between (7) and (8).

The bound applies to Dirac as well as Majorana transition moments. However, transition moments involving the electron and muon flavors are more strongly bounded by other accelerator experiments, $\kappa_{e} \lesssim 1.1 \times 10^{-9}, \kappa_{\mu} \lesssim 7.4 \times 10^{-9}$ [ 10$]$. Beam dump elastic scattering experiments also provide a bound on the tau neutrino diagonal moment which is more stringent by approximately an order of magnitude, $\kappa_{\tau} \lesssim 5.4 \times 10^{-7}$ [11]. A beam dump search for radiative decays gives a bound on the transition magnetic moment, $\mathcal{O}\left(10^{-9}\right)\left(\mathrm{MeV} / m_{\nu_{\tau}}\right)^{2}$, which is more stringent than (7) only for $m_{\nu_{\tau}} \gtrsim 10^{-2} \mathrm{MeV}$ [3]. 
Still more stringent bounds on neutrino magnetic moments $\mathcal{O}\left(10^{-(10-12)}\right)$ are available from astrophysical constraints [12], but these bounds apply only to neutrino masses which do not exceed stellar temperatures, $T \simeq \mathcal{O}(\mathrm{MeV})$. Furthermore, astrophysical constraints are "model dependent" in that they assume no new physics beyond the standard model [13].

The magnetic moment bound is relevant for ruling out $\mathrm{MeV}$ tau neutrinos since $\mathrm{MeV}$ neutrinos must decay rapidly to avoid nucleosynthesis constraints. In Refs. [3,4], it was pointed out that there is an open window for radiative decay into a sterile species. This window is further closed by the constraints discussed above.

T.M.G. would like to thank P. Fisher for useful discussions. The authors would like to acknowledge the hospitality of the ITP at Santa Barbara where some of this work was done.

\section{References}

[1] H. Grotch and R. Robinett, Z. Phys. C 39 (1988) 553.

[2] G.F. Giudice, Phys. Lett. B 251 (1990) 460.

[3] K.S. Babu, T.M. Gould and I.Z. Rothstein, Phys. Lett. B 321 (1994) 140.

[4] S. Dodelson, G. Gyuk and M. Turner, FERMILAB-Pub93236-A.

[5] L3 Collab., B. Adeva et al., Phys. Lett. B 252 (1990) 527.

[6] L3 Collab., O. Adriani et al., Phys. Lett. B 297 (1992) 471; Phys. Rep. 236 (1993) 1.

[7] ALEPH Collab., D. Decamp et al., Phys. Rep. 216 (1992) 253.

[8] T. Rizzo, Phys. Lett. B 237 (1990) 88.

[9] R. Escribano and E. Massó, Autònoma de Barcelona preprint UAB-FT-317, hep-ph/9403304.

[10] LAMPF Collab., D.A. Krakauer et al., Phys. Lett. B 252 (1990) 177; Phys. Rev. D 44 (1991) 6.

[11] A.M. Cooper-Sarkar et al., Phys. Lett. B 280 (1992) 153.

[12] G. Raffelt, Phys. Rev. Lett. 64 (1990) 2856;

G. Raffelt, D. Dearborn and J. Silk, Ap. J. 336 (1989) 61;

M. Fukugita and S. Yazaki, Phys. Rev. D 36 (1987) 3817; S. Nussinov and Y. Rephaeli, Phys. Rev. D 36 (1987) 2278; M.A.B. Beg, W. Marciano and M. Ruderman, Phys. Rev. D 17 (1978) 1395.

[13] K.S. Babu, R.N. Mohapatra and I.Z. Rothstein Phys. Rev. D 45 (1992) R3312. 